\title{
Vaccination Guidelines for Patients with Immune-mediated Disorders Taking Immunosuppressive Therapies: Executive Summary
}

\author{
Kim A. Papp, Boulos Haraoui, Deepali Kumar, John K. Marshall, Robert Bissonnette, \\ Alain Bitton, Brian Bressler, Melinda Gooderham, Vincent Ho, Shahin Jamal, Janet E. Pope, \\ A. Hillary Steinhart, Donald C. Vinh $\mathbb{D}^{\mathbb{D}}$, and John Wade
}

\begin{abstract}
The use of immunosuppressive therapies for immune-mediated disease is associated with an elevated risk of infections and related comorbidities. While many infectious diseases can generally be prevented by vaccines, immunization rates in this specific patient population remain suboptimal, due in part to uncertainty about their efficacy or safety under these clinical situations. To address this concern, a multidisciplinary group of Canadian physicians with expertise in dermatology, gastroenterology, infectious diseases, and rheumatology developed evidence-based clinical guidelines on vaccinations featuring 13 statements that are aimed at reducing the risk of preventable infections in individuals exposed to immunosuppressive and immunomodulatory agents. (First Release February 12019 ; J Rheumatol 2019;46:751-4; doi:10.3899/jrheum.180784)
\end{abstract}

Key Indexing Terms: VACCINATION

IMMUNOSUPPRESSION
IMMUNE-MEDIATED DISEASE
From K. Papp Clinical Research; Probity Medical Research, Waterloo; University Health Network; Faculty of Medicine, University of Toronto; Mount Sinai Hospital, Toronto; Department of Medicine and Farncombe Family Digestive Health Research Institute, McMaster University, Hamilton; Faculty of Medicine, Queen's University, Kingston; Faculty of Medicine, University of Western Ontario; St. Joseph's Health Care, London, Ontario; Centre Hospitalier de l'Université de Montréal; Innovaderm Research Inc.; McGill University Health Centre; Research Institute - McGill University Health Centre, Montreal, Quebec; Faculty of Medicine, University of British Columbia; St. Paul's Hospital; Vancouver Coastal Health; Vancouver General Hospital, Vancouver, British Columbia, Canada.

Development of the vaccination guidelines was funded by the Dermatology Association of Ontario. The authors received no financial support for the research, authorship, and/or publication of this article. K. Papp is an advisory board participant, steering committee member, investigator, speaker, and/or consultant for AbbVie, Amgen, Bristol-Myers Squibb, Celgene, Eli Lilly, Janssen, Merck (MSD), Novartis, and Valeant/Bausch Health. D. Kumar is an investigator for GSK, Oxford Immunotec, and Qiagen.J.K. Marshall is an advisory board participant, speaker, and/or consultant for AbbVie, Janssen, Pfizer, and Takeda. R. Bissonnette is an advisory board participant, investigator, speaker, and/or consultant for AbbVie, Amgen, Boehringer Ingelheim, Celgene, Eli Lilly, Galderma, Incyte, Janssen, and Pfizer, and is a shareholder of Innovaderm Research. A. Bitton is an advisory board participant, speaker, and/or investigator for AbbVie and Janssen. B. Bressler is an advisor and/or speaker for AbbVie, Allergan, Genentech, Janssen, Pfizer, and Takeda. M. Gooderham is an advisory board participant, investigator, consultant, and/or speaker for AbbVie, Amgen, Celgene, Eli Lilly, Galderma, Janssen, LEO Pharma, Novartis, Pfizer, Sanofi Genzyme, and Valeant Pharmaceuticals. S. Jamal is a consultant for BMS, Eli Lilly, and UCB, and has received research grants from Merck, AbbVie, and BMS. A.H. Steinhart is an advisory board participant, speaker, investigator, and/or consultant for AbbVie, Amgen, Arena Pharmaceuticals, Celgene, Ferring, Genentech, Hoffmann-La Roche, Hospira, Janssen, Merck, Pfizer, Pharmascience, Red Hill Biopharma, and Takeda.D.C. Vinh is an advisory board participant, speaker, consultant, and/or investigator for Avir Pharma, Cidara, CSL Behring Canada, Novartis Canada, and Shire Canada. J. Wade is an advisory board participant and consultant for AbbVie, Amgen, BMS, Celgene, Janssen, Lilly, Novartis, Roche, Sanofi, and UCB.
K.A. Papp, MD, PhD, FRCPC, K. Papp Clinical Research, and Probity Medical Research; B. Haraoui, MD, FRCPC, Centre Hospitalier de l'Université de Montréal; D. Kumar, MD, MSc, FRCPC, University Health Network, and Faculty of Medicine, University of Toronto; J.K. Marshall, MD, MSc, FRCPC, AGAF, Department of Medicine and Farncombe Family Digestive Health Research Institute, McMaster University; R. Bissonnette, MD, Innovaderm Research Inc.; A. Bitton, MD, FRCP, McGill University Health Centre; B. Bressler, MD, MS, FRCPC, Faculty of Medicine, University of British Columbia, and St. Paul's Hospital; M. Gooderham, MSc, MD, FRCPC, Probity Medical Research, and Faculty of Medicine, Queen's University; V. Ho, MD, FRCPC, Faculty of Medicine, University of British Columbia; S. Jamal, MD, FRCPC, MSc, Vancouver Coastal Health; J.E. Pope, MD, MPH, FRCPC, Faculty of Medicine, University of Western Ontario, and St. Joseph's Health Care; A.H. Steinhart, MD, FRCP $(C)$, Faculty of Medicine, University of Toronto, and Mount Sinai Hospital; D.C. Vinh, MD, McGill University Health Centre, and Research Institute - McGill University Health Centre; $J$. Wade, MD, FRCP $(C)$, Faculty of Medicine, University of British Columbia, and Vancouver General Hospital.

Address correspondence to Dr. K.A. Papp, K. Papp Clinical Research and Probity Medical Research, 135 \& 139 Union St. East, Waterloo, Ontario N2J 1C4, Canada.E-mail: kapapp@ probitymedical.com

Full Release Article. For details, see Reprints and Permissions at jrheum.org

Accepted for publication October 30, 2018.

The use of immunosuppressive therapies, including certain conventional synthetic disease-modifying antirheumatic drugs (DMARD), targeted synthetic DMARD, and biologics, has improved disease control and quality of life for patients with autoimmune and inflammatory diseases. However, because these treatments may attenuate protective immunity, some patients are potentially at an increased risk of developing common and opportunistic infections, complicated by higher rates of related morbidity and mortality than age- and sex-matched control populations s, $^{1,3,4}$. Although this risk can

Personal non-commercial use only. The Journal of Rheumatology Copyright @ 2019 . All rights reserved. 
be significantly reduced with commercially available vaccines, physicians often hesitate to vaccinate these patients because of uncertainties regarding the safety and efficacy of immunization while being treated with immunosuppressive medications $5,6,7,8,9$.

This executive summary of the clinical recommendations provides guidance regarding the vaccination of adults receiving immunosuppressive medications for the treatment of immune-mediated diseases (IMD), or infants with intrauterine exposure to such agents.

\section{MATERIALS AND METHODS}

A Canadian multidisciplinary committee with expertise in gastroenterology (JKM, AB, BB, AHS), dermatology (KAP, MG, RB, VH), rheumatology (BH, JEP, JW, SJ), and infectious diseases (DK, DCV) developed guidelines on the management of vaccination in patients receiving immunosuppressive therapies. Literature searches by Synapse Medical Communications identified clinical trials, metaanalyses, systematic reviews, observational studies, case series, and existing guidelines published from 2009 to 2017 across multiple databases (Embase, MEDLINE, PubMed) as per the Grading of Recommendation, Assessment, Development, and Evaluation (GRADE) system ${ }^{10}$. Reference lists were manually searched to identify relevant articles and included based on the committee's discretion (Figure 1). Published studies were then reviewed by the committee and assessed according to the GRADE evidence levels ${ }^{10}$. The quality of evidence was rated as "high" (indicating that further research is unlikely to change the confidence in the estimate of effect), "moderate" (implying that further research is likely to have an effect on the confidence in the estimate of effect), "low" (suggesting that further research is likely to have a strong effect on the confidence in the estimate of effect), or "very low" (meaning that any estimate of effect is very uncertain).

The Steering Committee [KAP (chair), JKM, DK, BH] developed the initial statements, which underwent 2 rounds of revisions according to feedback received from all authors. All 14 members voted on a Web-based

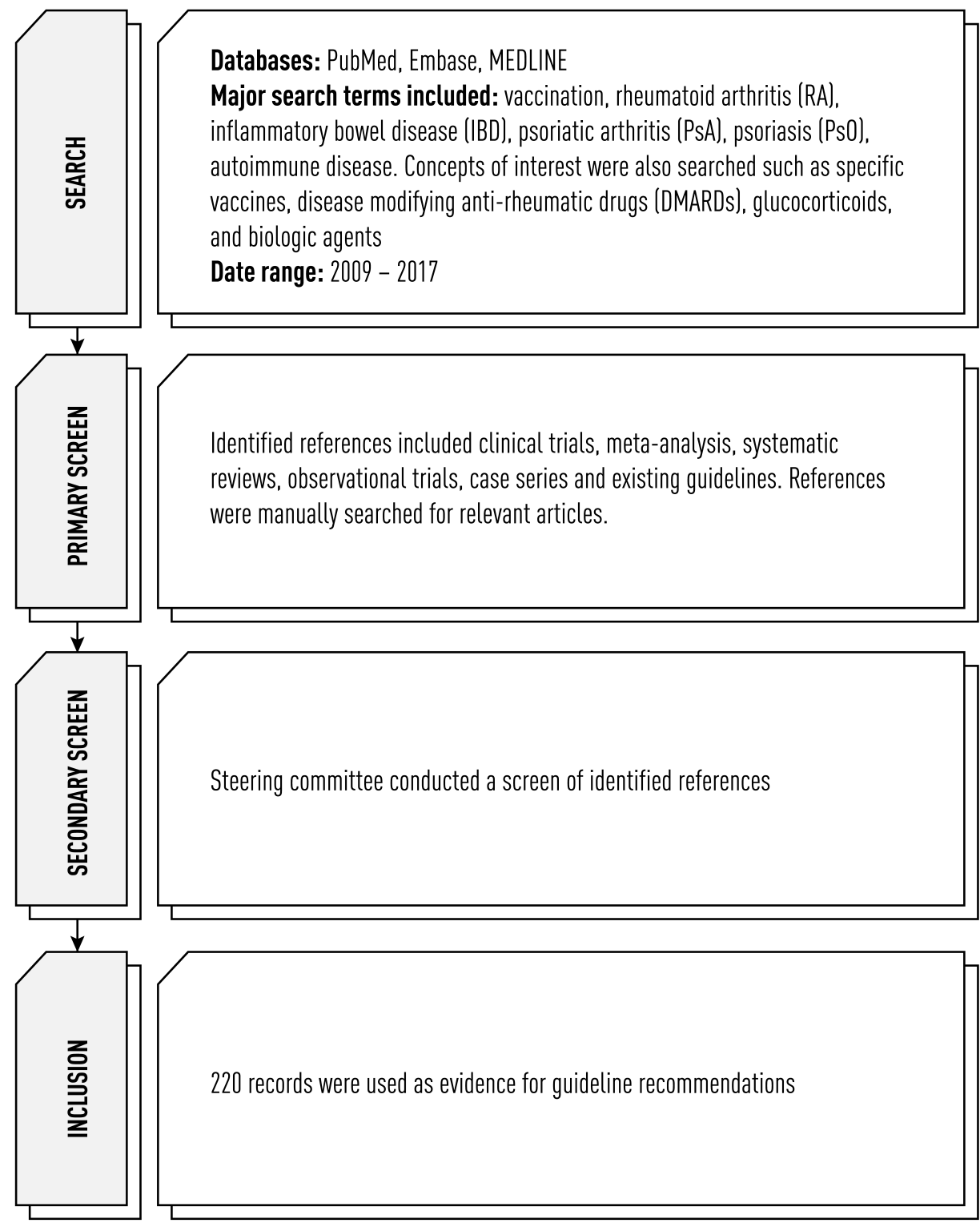

Figure 1. Literature search methodology. Personal non-commercial use only. The Journal of Rheumatology Copyright (c) 2019. All rights reserved. 
platform to determine the level of agreement for each statement using a 5-point scale (strongly agree, agree, neutral, disagree, strongly disagree). Statements achieving $\geq 75 \%$ agreement were included in the guidelines. Of the 15 statements considered, 2 were rejected.

The strength of recommendations was evaluated according to the
GRADE and rated as "strong" when desirable consequences clearly outweighed undesirable consequences, "conditional" when desirable consequences probably outweighed undesirable consequences, or "weak" when the balance between desirable and undesirable consequences was closely balanced or uncertain

Table 1. Guideline statements*.

Statement

Recommendation

Statement 1

Inactivated vaccines

Statement $2 \mathrm{a}$

Statement $2 b$

Statement $2 c$

Live attenuated herpes zoster vaccine

Statement 3a To optimize the immunogenicity of the live attenuated herpes zoster
vaccine in treatment-naive patients with immune-mediated conditions, we suggest immunization be performed at least 2-4 weeks prior to initiation of immunosuppressive therapy.

Statement $3 b$ In patients with immune-mediated diseases receiving immunosuppressive agents, the live attenuated herpes zoster vaccine can be safely administered to patients at risk, but the subunit vaccine is the preferred alternative.

Individual situations should be assessed for patients treated with a combination of immunosuppressive drugs, if the live vaccine is being considered.

Other live attenuated vaccines

Statement $4 \mathrm{a}$

In treatment-naive patients with immune-mediated diseases who are vaccinated with live attenuated vaccines, we recommend that the duration of viremia following immunization be considered when determining the optimal time to initiate immunosuppressive therapy.

Statement $4 b$ In patients with immune-mediated diseases who interrupt immunosuppressive treatment prior to vaccination, we recommend that the duration of viremia following immunization be considered when determining the optimal time to re-initiate immunosuppressive therapy.

Statement $4 \mathrm{c}$ In patients with immune-mediated diseases receiving immunosuppressive agents, we suggest that live attenuated vaccines be administered when individual benefits outweigh the perceived risks.

Statement $4 d$ In situations where patient safety is a paramount concern and the clinical situation allows, we suggest that immunosuppressive treatment be interrupted for a duration based on drug pharmacokinetics prior to immunization with live vaccines.

Vaccination of infants with early exposure to immunosuppressive agents

Statement 5a In infants exposed to immunosuppressive agents in utero during the third trimester, we recommend that inactivated vaccines be administered according to the local immunization schedule.

Statement $5 b$ In infants exposed to immunosuppressive agents in utero during the third trimester, we recommend that the MMR and varicella vaccines be administered according to the local immunization schedule.

Statement $5 \mathrm{c}$ In infants breast-fed by mothers receiving immunosuppressive regimens, we recommend that inactivated and live attenuated vaccines be administered according to the local immunization schedule without delay.
Evidence Level/Recommendation Strength

Strong recommendation, moderate-level evidence

Conditional recommendation, moderate-level evidence

Strong recommendation, moderate-level evidence

Strong recommendation, low-level evidence

Conditional recommendation, moderate-level evidence

Strong recommendation, moderate-level evidence

Strong recommendation, very low-level evidence

Strong recommendation, very low-level evidence

Conditional recommendation, low-level evidence

Conditional recommendation, low-level evidence

Strong recommendation, very low-level evidence

Strong recommendation, low-level evidence

Strong recommendation, very low-level evidence

* Further information is available in the full guidelines ${ }^{11}$. RTX: rituximab; MMR: measles, mumps, rubella. 


\section{RESULTS}

The developed guidelines consist of 13 statements addressing general immunization strategies for individuals exposed to biologic and/or nonbiologic immunomodulatory agents (Table 1) ${ }^{11}$. Of these, 10 statements focus on the management of adults with IMD who are considering age-appropriate primary and secondary immunizations with live or inactivated vaccines. Recommendations specifically regarding the use of the live attenuated herpes zoster vaccine are also provided. The remaining 3 statements pertain to the timing of routine childhood vaccinations in infants exposed to immunosuppressive drugs either in utero during the third trimester or through breastfeeding.

In the full guideline document, each statement is followed by a discussion of the supporting evidence, as well as any existing recommendations or guidance from other physician $\operatorname{organizations}_{\text {or societies }}{ }^{11}$.

\section{DISCUSSION}

This document is intended to provide guidance on the vaccination of individuals exposed to immunosuppressive therapies. A robust discussion of these recommendations is provided in the full guideline document ${ }^{11}$.

These guidelines were developed according to the best data available to date. However, the body of evidence regarding the safety and efficacy of vaccination in this patient population is small and incomplete. Therefore, clinical judgment based on a careful assessment of patient factors and the risks and benefits of vaccination should always prevail when determining the best course of action for each individual.

Regular updates to the current guidelines will be necessary as new clinical trial data and treatment options emerge.

\section{ACKNOWLEDGMENT}

We thank Synapse Medical Communications, Oakville, Ontario, Canada, for providing medical writing assistance in the form of drafting and revising as per authors' directions and in accordance with the standards set out by the International Committee of Medical Journal Editors. Medical writing support was funded by the Dermatology Association of Ontario.

\section{REFERENCES}

1. Yun H, Yang S, Chen L, Xie F, Winthrop K, Baddley JW, et al. Risk of herpes zoster in autoimmune and inflammatory diseases: implications for vaccination. Arthritis Rheumatol 2016;68:2328-37.

2. Shigayeva A, Rudnick W, Green K, Chen DK, Demczuk W, Gold WL, et al; Toronto Invasive Bacterial Diseases Network. Invasive pneumococcal disease among immunocompromised persons: implications for vaccination programs. Clin Infect Dis 2016; 62:139-47.

3. Doran MF, Crowson CS, Pond GR, O'Fallon WM, Gabriel SE. Frequency of infection in patients with rheumatoid arthritis compared with controls: a population-based study. Arthritis Rheum 2002;46:2287-93.

4. McKinnon JE, Maksimowicz-McKinnon K. Autoimmune disease and vaccination: impact on infectious disease prevention and a look at future applications. Transl Res 2016;167:46-60.

5. Assala M, Groh M, Blanche P, Vinter C, Cohen P, Le Guern V, et al. Pneumococcal and influenza vaccination rates in patients treated with corticosteroids and/or immunosuppressive therapies for systemic autoimmune diseases: a cross-sectional study. Joint Bone Spine 2017;84:365-6.

6. Lawson EF, Trupin L, Yelin EH, Yazdany J. Reasons for failure to receive pneumococcal and influenza vaccinations among immunosuppressed patients with systemic lupus erythematosus. Semin Arthritis Rheum 2015;44:666-71.

7. Hmamouchi I, Winthrop K, Launay O, Dougados M. Low rate of influenza and pneumococcal vaccine coverage in rheumatoid arthritis: data from the international COMORA cohort. Vaccine 2015;33:1446-52.

8. Loubet P, Kernéis S, Groh M, Loulergue P, Blanche P, Verger P, et al. Attitude, knowledge and factors associated with influenza and pneumococcal vaccine uptake in a large cohort of patients with secondary immune deficiency. Vaccine 2015;33:3703-8.

9. Hua C, Morel J, Ardouin E, Ricard E, Foret J, Mathieu S, et al. Reasons for non-vaccination in French rheumatoid arthritis and spondyloarthritis patients. Rheumatology 2015;54:748-50.

10. Guyatt GH, Oxman AD, Vist GE, Kunz R, Falck-Ytter Y, Alonso-Coello P, et al; GRADE Working Group. GRADE: an emerging consensus on rating quality of evidence and strength of recommendations. BMJ 2008;336:924-6.

11. Papp KA, Haraoui B, Kumar D, Marshall JK, Bissonnette R, Bitton $\mathrm{A}$, et al. Vaccination guidelines for patients with immune-mediated disorders on immunosuppressive therapies. J Cutan Med Surg 2018 Nov 21 (E-pub ahead of print). 